\title{
Pharmacy travel health services: current perspectives and future prospects
}

This article was published in the following Dove Press journal: Integrated Pharmacy Research and Practice

\section{Sherilyn KD Houle}

School of Pharmacy, University of Waterloo, Waterloo, ON, Canada
Correspondence: Sherilyn KD Houle School of Pharmacy, University of Waterloo, 200 University Avenue West, Waterloo, ON N2L 3GI, Canada $\mathrm{Tel}+\mathrm{I} 5198884567$ (ext 21378) $\mathrm{Fax}+$ I 5197425322

Email sherilyn.houle@uwaterloo.ca

\begin{abstract}
Rates of international travel are increasing annually, with particular growth observed in travel to Southeast Asia and to emerging economies. While all patients traveling across geographic regions are recommended to receive a pre-travel consultation to consider their individual risks, many do not, or receive care and recommendations that are not consistent with current evidence-based guidelines. As experts in drug therapy, and given the largely preventive nature of most travel health recommendations, pharmacists are well suited to help address this need. Pharmacists generally possess a high degree of knowledge and confidence with more commonly observed travel health topics in community practice such as travelers' diarrhea; however, training in more specialized travel health topics such as travel vaccinations and traveling at altitude has generally been lacking from pharmacy curricula. Pharmacists with an interest in providing pre-travel consultations are encouraged to pursue additional training in this specialty and to consider Certificate in Travel Health designation from the International Society of Travel Medicine. Future roles for pharmacists to include the prescribing of medications and vaccines for travel and the in-pharmacy administration of travel vaccinations may improve patient access to pretravel consultations and recommended preventive measures, improving the health of travelers and potentially reducing the burden of communicable disease worldwide. Pharmacists providing travel care to patients are also reminded to consider noninfectious risks of illness and injury abroad and to counsel patients on strategies to minimize these risks in addition to providing drug and vaccine recommendations.
\end{abstract}

Keywords: pharmacist, community pharmacy, travel, vaccination

\section{Introduction}

The United Nations World Tourism Organization has reported a steady rise in international travel. In fact, 2016 marked the 7th consecutive year of above-average international arrivals, reaching 1.2 billion. ${ }^{1}$ This is expected to continue to increase at a rate of $3.3 \%$ annually through $2030 .^{2}$

\section{The importance of evidence-based pre-travel consultations}

The World Health Organization, ${ }^{3}$ Centers for Disease Control and Prevention, ${ }^{4}$ the Public Health Agency of Canada, ${ }^{5}$ and others recommend that individuals crossing international boundaries receive a pre-travel consultation. According to Hatz and Chen, the pre-travel consultation should fulfill the following 3 goals: 1) assessment of the client's fitness for travel, based on their medical history and an understanding of the purpose and type of travel; 2) analysis of the anticipated and real health risks; 
and 3) translation of the findings into a tailored counseling of prophylactic measures. ${ }^{6}$ Both infectious and noninfectious risks must be considered, with consultations tailored to consider patient-specific risks as well as destination- and itinerary-specific risks. $^{7}$

Despite the importance of these preventive measures, evidence suggests that few travelers receive evidence-based care, if they receive a pre-travel consultation at all. Research among travelers in airports estimated that $22-86 \%$ had sought some form of travel health advice prior to their departure; however, internet-based resources were consulted at the same or higher frequency than health professionals. ${ }^{8-11}$ Additionally, the relatively small sample sizes of these studies (range 262-5,465 travelers) in comparison to the overall population of international travelers may affect the generalizability of these findings. A Canadian study of 1,724 air passengers reported that fewer than 1 in 10 individuals traveling to Mexico or the Dominican Republic received a travel health consultation. ${ }^{12}$ Other research studies considering the quality of travel health advice provided by North American practitioners concluded that while advice on travelers' diarrhea was generally of high quality, 20-75\% of immunizations recommended were inadequate or inappropriate, and $40 \%$ gave incorrect recommendations for antimalarial prophylaxis based on responses to 5 hypothetical cases. ${ }^{13}$

Survey research suggests that $22-64 \%$ of travelers experience some degree of health impairment while traveling. ${ }^{14-16}$ Travelers' diarrhea is most common, affecting 30-80\% of travelers, depending on the destination, with malaria and vaccine-preventable infections significantly less common. ${ }^{17}$ A study of fatalities among French travelers from 2000-2004 highlighted the often noninfectious causes of death while traveling. In this study, only $1.4 \%$ of deaths were infectionrelated, with the highest proportion of deaths due to traffic accidents (28.1\%) and cardiovascular disease (27.4\%). ${ }^{18}$ For this reason, pre-travel consultations should not be limited only to vaccines and pharmacotherapy as preventive measures, but should also include the management of pre-existing conditions, traffic safety, and safe sexual practices, among others. However, the need for vaccinations and prescription drugs can serve as a driver to encourage patients to visit their health care provider where these related discussions on other travel health risks can be held.

\section{Pharmacists' contact with travelers}

Pharmacies are one of the most accessible health service settings worldwide, located in rural and urban areas, and often with longer operating hours than medical and public health clinics. In addition to counseling patients presenting with prescriptions of drugs for travel purposes (e.g., antimalarials, altitude sickness prophylaxis, travelers' diarrhea treatment), pharmacists are also a key point of contact for non-prescription product advice such as for anti-diarrheal, insect repellant, and sunscreen products. A survey of 53 community pharmacists in Alberta, Canada, in 2012 found that 1 in 4 respondents reported counseling a patient on a travel-related topic at least once per week, increasing to $69 \%$ of respondents doing so at least monthly. ${ }^{19}$ As international travel rates continue to grow, so too will opportunities for pharmacists to interact with individuals who are planning travel.

As most recommendations and treatments arising from pre-travel consultations are for illness and injury prevention, diagnostic skills are not often required. Pharmacists' expertise in drug therapy and vaccinations, and high level of public trust and accessibility create a unique opportunity for pharmacists to help improve the number and quality of pre-travel consultations received by travelers.

\section{Current perspectives The administration of injections by pharmacists}

As a result of their long-standing role in dispensing injections and other storage-sensitive products, pharmacies are already well equipped to ensure cold chain protection for vaccinations. Prior to pharmacists beginning to adopt a role in the administration of these products, the dispensed product was often taken home by the patient for injection by a physician or nurse at a later time. This may result in a delay in receiving the injection as well as potentially compromised stability if the product was improperly stored between dispensing and injection. To address this issue and to encourage greater vaccination rates at the population level, the United States introduced legislation allowing for pharmacist administration of injections. By 2009, all 50 states had some form of injection authorization in place. ${ }^{20}$ Canada, ${ }^{21}$ the United Kingdom, ${ }^{22}$ Australia, ${ }^{23}$ and Portugal, ${ }^{24}$ among others, have also expanded the scope of pharmacists to include injection administration. Vaccination against influenza has been the most common addition to the pharmacist's scope in many regions, although broader legislation allowing the administration of other vaccines, including travel vaccines, has been adopted in some jurisdictions. Other jurisdictions allow for the injection of non-influenza vaccines by pharmacists under a medical directive.

Pharmacist-administered vaccinations have been found to be well-received, including high patient satisfaction with 
the service, ${ }^{25,26}$ patient recognition of the greater accessibility of vaccination through pharmacies than through other care settings ${ }^{25}$ and improvements in vaccination rates following the introduction of pharmacist-administered injections. ${ }^{27-29}$ While many of these studies focused on influenza and other routine vaccinations, the administration of vaccinations for travel purposes is unlikely to present major changes to established practice for pharmacists who administer other vaccinations. Training to administer vaccinations includes landmarking and administration via both the intramuscular and subcutaneous routes, assessment for the appropriateness of live versus inactivated vaccines, and monitoring and treatment of anaphylaxis and other vaccination-related reactions, which is consistent across all vaccines. When presented with an order from a prescriber for these vaccinations for a patient following a pre-travel consultation, the administration process and workflow is expected to be similar to non-travel vaccines. However, pharmacists may have learning needs related to assessing the appropriateness of vaccines when this is based on itinerary-specific or destination-specific risks, or weighing unique patient risks associated with a particular travel vaccine, such as vaccine-associated neurotropic or viscerotropic disease with yellow fever vaccination among older travelers. Pharmacists may opt to refer these more complicated cases to specialty travel clinics for assessment and education.

\section{Pharmacist prescribing}

Pharmacists have long been providing patients with nonprescription drug therapies for travelers' diarrhea, motion sickness, sun protection, and insect bite protection. However, patients requiring prescription drug therapies traditionally had to be referred to their family physician or a travel clinic for these orders. Pharmacist prescribing can take many forms. ${ }^{30}$ "Independent prescribing" occurs when the health professional making the prescribing decision possesses the knowledge and skills to assess the condition being treated and takes sole responsibility for the prescription issued and resulting patient outcomes. "Dependent (or delegated) prescribing" is when authority to prescribe, often following a pre-defined protocol, is granted from a professional with prescribing authority to another health professional who otherwise would not have prescribing authority. Formal agreements between the professionals must exist specifically outlining the scope that this prescribing activity can encompass. Finally, "collaborative prescribing" is also defined by an agreement between a prescriber and another health professional; however, these agreements do not specify protocols that must strictly be adhered to, but rather identify a particular patient population that the pharmacist can prescribe for based on their professional judgment.

Two studies to date of pharmacist-performed consultations have utilized a recommendation-based approach, with these recommendations communicated to a prescriber (in both studies, a physician) who is then responsible for the prescribing decision. A 2015 study by Tran et $\mathrm{al}^{31}$ reported the results of a telephone survey of 103 patients who had received a pre-travel consultation from a supermarket-based community pharmacist. This pharmacist possessed Certificate in Travel Health (CTH) designation from the International Society of Travel Medicine (ISTM), yet required a physician's order for prescription of drugs and some vaccines. This study found that $79 \%$ of patients recommended an antimalarial drug ultimately obtained it, and $42 \%$ of patients recommended acetazolamide for the prevention of altitude sickness obtained it before their trip. It was unclear what proportion of patients not receiving the recommended prescription drugs did so out of personal choice, or because they were unable to obtain a prescription from their physician. A study by Jackson et al described a pharmacist-run travel consultation service that largely operates via telephone communication with patients. ${ }^{32}$ The clinic team consists of an infectious diseases physician, infectious diseases pharmacy specialist, staff pharmacists, and a pharmacy technician. In this clinic, the pharmacists provided recommendations to the in-clinic infectious diseases physician for approval. This paper compared the recommendations of pharmacists at this clinic to those of a nurse-based travel clinic, and estimated that the pharmacist-provided care could save approximately USD $\$ 47,000$ annually as a result of more appropriate prescribing of vaccines and medications, assuming a rate of 720 consultations per year.

A report by Hess et $\mathrm{a}^{33}$ examined pharmacist prescribing by collaborative practice protocol at an independent community pharmacy in California. This pharmacist also had CTH designation. According to the 283 patients surveyed, $85 \%$ of the pharmacist's recommendations were accepted overall, ranging from $67 \%$ for polio vaccination to $94 \%$ for antimalarial drugs and $97 \%$ for yellow fever vaccination, with low perceived risk of contracting the disease as the most commonly cited reason for refusal.

The patient satisfaction and clinical outcomes of independent prescribing by a pharmacist in Alberta, Canada, has recently been studied. This pharmacist also has CTH designation and has independent prescribing authorization for all vaccines and medications needed for travel purposes. Chart 
review performed on 103 patients seen in the clinic identified that $79 \%$ of recommended vaccinations were administered in clinic, with rabies and Japanese encephalitis the most frequently declined. Of the 78 patients who completed a post-travel survey on their experience at the clinic and health status while traveling, 94\% reported being satisfied or very satisfied with the service received. Fifteen patients reported experiencing a health issue abroad, most commonly travellers' diarrhea, and felt well prepared to manage these issues based on the advice received during the consultation. ${ }^{34}$

\section{Pharmacist knowledge and confidence in travel health}

As travel health is a relatively new field (first formally defined in 1988$)^{35}$ and pre-travel consultations and vaccinations have until recently been largely performed by primary care physicians and physician- or nurse-operated travel clinics, few pharmacy programs have included travel health in their curricula. As a result, pharmacists entering the profession a decade or more ago likely had minimal formal training in travel-related topics beyond non-prescription drug recommendations. While this has not yet been systematically measured on a large scale, lack of knowledge or confidence in travel health has been cited as a barrier preventing the greater provision of travel vaccination services in community pharmacy practice. ${ }^{36}$

The survey of community pharmacists in Alberta, Canada, mentioned previously, ${ }^{19}$ asked respondents to answer multiple-choice questions related to 2 patient care cases, addressing knowledge on topics including travelers' diarrhea, altitude sickness, malaria, vaccinations, and other preventable health risks. Respondents were asked to answer the questions to the best of their ability without consulting any clinical references, and were also provided the option of "I don't know, I would have to look this up" when they were uncertain. They were then asked to rate their level of confidence in the answers they had provided. These pharmacists scored most accurately on questions related to the steps involved in a pretravel assessment (98\% of respondents selected the correct answer) and travelers' diarrhea (40\%), and most poorly on questions related to knowledge of vaccines (4\%) and travel at altitude $(2 \%)$. Confidence in each area of knowledge followed a similar pattern, with overall confidence rated by $21 \%$ as "confident", $8 \%$ as "not confident", and the remaining $71 \%$ as "neutral". While this reflects a general lack of confidence in providing travel care unaided, two-thirds reported that they had confidence in their ability to locate the required clinical information using various references.
It is not surprising that pharmacists were both most knowledgeable and confident in topics they are most likely to encounter in community pharmacy practice, with declining knowledge and confidence in more specialized topics that may not have been part of their formal training. This highlights the need for educational programs to be developed to support practicing pharmacists in acquiring the knowledge and skills needed to embrace these new practice opportunities in travel health, and the integration of travel health into the curricula of entry-to-practice degree programs.

\section{Future prospects Growing demand for pharmacist training in travel health}

As previously mentioned, the rate of international travel is expected to continue to grow through $2030 ;{ }^{1}$ therefore, pharmacists can expect to be more frequently approached for advice and care by travelers. While pharmacists feel most confident in their ability to provide advice related to travelers' diarrhea and other non-prescription products such as sunscreens and anti-emetics, increasing rates of travel are specifically predicted to Southeast Asia and emerging economies such as Latin America and Africa. ${ }^{1}$ Travel to these regions requires consideration of other disease risks such as yellow fever, malaria, and Japanese encephalitis. It is therefore expected that the demand for additional knowledge and training by pharmacists in travel health will increase to meet these needs. Continuing professional development programs developed at the local, national, and international levels are predicted to increase in frequency, coupled with an increasing rate of pharmacists seeking their CTH designation to recognize this expertise. ${ }^{37}$ In 2009 , ISTM established a Pharmacist Professional Group specifically to represent pharmacists around the world who practice in travel medicine, allowing members to share their knowledge and expertise with other pharmacists.

\section{Consideration of adding travelers' diarrhea and travel vaccines to minor ailment pharmacist prescribing programs}

A number of regions across $\mathrm{Canada}^{38}$ and the United Kingdom $^{39}$ authorize pharmacists to issue prescriptions for minor (also referred to as "common") ailments. Minor ailments have been defined as "common or self-limiting or uncomplicated [conditions] that may be diagnosed and managed without medical intervention". ${ }^{40}$ As such, one could argue that the issuing of a prescription for antibiotic therapy for 
the treatment of travelers' diarrhea to a patient with upcoming travel plans could be considered a qualifying minor ailment, as physical assessment and other diagnostic exams are not required for determining the appropriateness of the prescription. The condition is common among travelers, and it is generally self-limiting. Pharmacists dispensing these antibiotics already counsel patients on food and water precautions, when to initiate treatment, and when to seek medical attention; therefore, one can expect patients to receive safe and effective prescribing of these agents by their pharmacist.

\section{Expansion of prescribing and vaccination authority}

While minor ailment programs like those mentioned earlier are generally widely applicable to most practicing pharmacists, with prescribing limited to specific conditions and treatment protocols, other regions have adopted broader independent prescribing programs that often require an additional qualification or demonstrated knowledge in certain prescribing areas.

One of the broadest programs, termed Additional Prescribing Authorization, is offered to pharmacists in Alberta, Canada, with at least 1 year of practice experience. ${ }^{41}$ In order to receive this designation, pharmacists must demonstrate competence in therapeutic area(s) in which they anticipate applying their prescribing authorization (if successful), by providing evidence of completed continuing education in that field and submitting actual patient care cases encountered in their practice. These cases are assessed by the provincial regulatory body and pharmacist reviewers for evidence of clinical competence, documentation, follow-up and monitoring, effective collaboration with other clinicians, acknowledgement of areas of strength and situations where referral to other health professionals would be warranted, and other considerations to ensure the safe and effective application of this expanded scope service. Pharmacists who are successful in their application can prescribe any drug - with the exception of narcotics, benzodiazepines, and other controlled substances - provided that they obtain patient consent to prescribe and restrict their prescribing only to those cases where they feel they can safely and competently prescribe. Pharmacists are therefore required to apply their professional judgment to each case to determine if prescribing for that patient would be appropriate and do not need to adhere to pre-specified prescribing protocols, as this represents a form of independent prescribing. Pharmacists in Alberta with an interest in travel health can therefore acquire additional knowledge in travel health, obtain this authorization, and use it to provide complete pre-travel consultations for patients including the issuing of prescriptions for needed drugs and vaccines.

Pharmacists in Manitoba, Canada, with board certification in a clinical specialty (or equivalent) or a post-baccalaureate Pharm.D. credential coupled with a specified minimum number of hours of practicing in that area, can apply for Extended Practice Pharmacist designation, ${ }^{42}$ which provides independent prescriptive authority to the pharmacist for that clinical specialty. However, to qualify for this designation, pharmacists must practice in a "collaborative practice setting", which is defined as one in which the pharmacist:

"works closely and collaboratively with other health care professionals (physicians and/or nurse practitioners) to provide care to a common patient or group of patients $[\ldots]$ [and] have access to the same diagnostic and health information to aid in providing enhanced care to the patient."

While potentially not as inclusive of community pharmacist practitioners as the Alberta legislation, pharmacists working in close collaboration with a primary care clinic and access to the same electronic medical record with specialty credentialing in travel health could apply this legislation toward obtaining similar prescribing rights for patients who are traveling.

Expansion in the pharmacist's scope of practice to include prescribing, coupled with the authorization to administer a broad range of vaccines, will best allow pharmacists to provide accessible and evidence-based care to travelers. Most pharmacist vaccination programs worldwide were developed with the initial goal of improving vaccination rates for influenza, and a number of regions restrict pharmacistadministered vaccinations to the influenza vaccine, while others provide a restricted list of vaccines that often focus on routine vaccines and exclude those for travel purposes. ${ }^{43}$ Apart from the yellow fever vaccine which, as a live vaccine, requires careful consideration of its timing in relation to other vaccines and is contraindicated in immunocompromised patients, ${ }^{44}$ no additional risks are anticipated by pharmacists administering travel vaccinations when compared to influenza vaccinations. Even in the absence of pharmacist prescribing authority for vaccines requiring a prescription, the ability for patients to receive the injection at their pharmacy has the potential to improve adherence to multiple-dose vaccine regimens due to the greater accessibility and convenience of pharmacies when compared to other primary care centers. Additionally, the stability of storage-sensitive vaccines can be more confidently assured when the vaccine is administered at the point of dispensing when compared to being taken home by the patient for injection at a later time (in situations where 
vaccines are not dispensed by the travel clinic or prescriber). With the key goal of most vaccination programs being to increase population vaccination rates, any efforts to improve patient convenience and accessibility should be encouraged.

\section{Pharmacists supporting immunization registries and other documentation efforts}

Despite technologic advances, many pharmacists worldwide lack access to an electronic health record that is viewable by all health professionals providing care for patients. Vaccination records are often stored within public health departments or individual practices. For example, a vaccine administered at a public health clinic may only be documented in their internal records, with similar practices in medical clinics and pharmacies. As a result, determination of an individual patient's vaccination history can be challenging as each provider must be contacted for their records, and population vaccination rates can be difficult to estimate. An immunization registry is defined as "an electronic system that supports the centralized storage and retrieval of immunization events and patient immunization profiles" 45 and may offer a solution to the current fragmented system of immunization records. As pharmacies have well-established documentation systems for the dispensing of drugs and vaccines, and as pharmacists are increasingly granted authorization to administer a range of vaccines, it is important for the pharmacy profession to be involved in the development of immunization registries. In the absence of region- or nation-wide registries, pharmacists can also play a role in supporting patients in maintaining documentation of their vaccination history, potentially aided by the use of a number of smartphone apps and other software programs developed for this purpose. ${ }^{46}$

\section{Enhanced focus of counseling efforts on noninfectious causes of morbidity and mortality among travelers}

While vaccinations and oral therapies for the prevention of infectious diseases are often considered a cornerstone of pre-travel care, noninfectious causes of illness and injury are more prevalent among travelers and require greater attention during travel consultations. Road traffic accidents, thrombosis, and exacerbations of existing chronic illnesses, for example, are more likely to contribute to death abroad than infectious disease. ${ }^{47-49}$ Pharmacists must ensure that travel consultations are comprehensive and consider all potential risks the traveler may face, rather than a narrow focus on vac- cinations and non-vaccine-preventable infectious diseases. Within the increasingly busy environment of community pharmacy practice, pharmacists are strongly encouraged to allocate dedicated time for travel consultations (preferably utilizing an appointment-based system to ensure adequate staffing of the pharmacy) performed in a private consultation room, and to charge patients for their time and expertise when providing a comprehensive pre-travel consultation. This protected and uninterrupted time with patients can help ensure that these consultations offer sufficient time to address all important travel health considerations. Charging patients for the consultation disconnects the viability of the service from the dispensing of prescriptions or the sale of non-prescription drugs, allowing pharmacists to ensure that even previously vaccinated travelers or those at risk of nonmedical risks abroad receive comprehensive care.

\section{Conclusion}

Pharmacists are a frequent point of contact for patients with plans to travel as providers of non-prescription drug advice, dispensing of prescription drugs and vaccines for travel purposes, and the administration of vaccines in some jurisdictions. Increasing rates of international travel and evidence that not all patients who would benefit from a pre-travel consultation receive one create an opportunity for pharmacists to help fill this care gap. Expansion of prescriptive authority to include treatment for travelers' diarrhea, travel vaccines, and other necessary preventive and therapeutic care will further support the role of pharmacists as travel health care providers.

\section{Disclosure}

SKDH has been a member of an expert consultant panel for Valneva Canada. The author reports no other conflicts of interest in this work.

\section{References}

1. World Tourism Organization. UNWTO Annual Report 2016. Madrid: UNWTO; 2017.

2. Glaesser D, Kester J, Paulose H, et al. Global travel patterns: an overview. J Trav Med. 2017;24(4):1-5.

3. World Health Organization. International Travel and Health - General Precautions. Available from: http://www.who.int/ith/precautions/en/. Accessed October 25, 2017.

4. Centres for Disease Control and Prevention. See a Doctor Before you Travel. Available from: https://wwwnc.cdc.gov/travel/page/see-doctor. Accessed October 25, 2017.

5. Government of Canada. Travel health and safety. Available from: https:// travel.gc.ca/travelling/health-safety?_ga=1.194135083.70670169.1455 904591. Accessed October 25, 2017.

6. Hatz C, Chen LH. Pre-travel consultation. In: Keystone JS, Freedman DO, Kozarsky P, Nothdurft HD, Connor BA, editors. Travel Medicine. 3rd ed. Elsevier; 2013:31-36. 
7. Aw B, Boraston S, Botten D, et al. Travel medicine: What's involved? When to refer? Can Fam Phys. 2014;60(12):1091-1103.

8. Van Herck K, Castelli F, Zuckerman J, et al. Knowledge, attitudes and practices in travel-related infectious diseases: the European Airport Survey. J Trav Med. 2004;11(1):3-8.

9. Hamer DH, Connor BA. Travel health knowledge, attitudes and practices among United States travelers. J Trav Med. 2004;11(1):23-26.

10. Toovey S, Jamieson A, Holloway M. Travellers knowledge, attitudes and practices on the prevention of infectious diseases: results from a study at Johannesburg International Airport. J Trav Med. 2004;11(1): $16-22$.

11. Al-Abri SS, Abdel-Hady DM, Al-Abaidani IS. Knowledge, attitudes, and practices regarding travel health among Muscat International Airport travelers in Oman: identifying the gaps and addressing the challenges. J Epidemiol Glob Health. 2016;6(2):67-75.

12. Provost S, Soto JC. Perception and knowledge about some infectious diseases among travelers from Québec, Canada. J Travel Med. 2002; 9(4):184-189

13. Keystone JS, Dismukes R, Sawyer L, Kozarsky PE. Inadequacies in health recommendations provided for international travelers by North American travel health advisors. J Trav Med. 1994;1(2):72-78.

14. Peltola H, Kyronseppa H, Holsa P. Trips to the South - a health hazard: morbidity of Finnish travelers. Scand J Infect Dis. 1983; 15(4):375-381.

15. Hill DR. Health problems in a large cohort of Americans traveling to developing countries. J Trav Med. 2000;7(5):259-266.

16. Reed JM, McIntosh IB, Powers K. Travel illness and the family practitioner: a retrospective assessment of travel-induced illness in general practice and the effect of a travel illness clinic. J Trav Med. 1994; 1(4):192-198.

17. Steffen R, deBernardis C, Banos A. Travel epidemiology - a global perspective. Int J Antimicrob Agents. 2003; 21(2):89-95.

18. Jeannel $\mathrm{D}$, Allain-Ioos $\mathrm{S}$, Bonmarin I, et al. Les décès de français lors d 'un séjour à l'étranger et leurs causes [The deaths of French citizens during a stay abroad and their causes]. Bull Epid Heb. 2006: 166-168. French.

19. Bascom CS, Rosenthal MM, Houle SKD. Are pharmacists ready for a greater role in travel health? An evaluation of the knowledge and confidence in providing travel health advice of pharmacists practicing in a community pharmacy chain in Alberta, Canada. J Trav Med. 2015;22(2):99-104.

20. Traynor K. With Maine on board, pharmacists in all 50 states can vaccinate: H1N1 prompts emergency vaccination rules for pharmacists. $A m$ J Health Sys Pharm. 2009;66(21):1892-1894.

21. Canadian Pharmacists Association. Pharmacists' expanded scope of practice. Available from: http://www.pharmacists.ca/index.cfm/ pharmacy-in-canada/scope-of-practice-canada/. Accessed October 25, 2017.

22. Pharmaceutical Services Negotiating Committee. Flu vaccination service. Available from: http://psnc.org.uk/services-commissioning/ advanced-services/flu-vaccination-service/. Accessed October 25, 2017.

23. Paola S. Pharmacist vaccination: where we are now. Australian Journal of Pharmacy online. Available from: https://ajp.com.au/news/ pharmacist-vaccination-now/. Accessed October 25, 2017.

24. Dias de Almeida P. Flu vaccination in pharmacies in Portugal. Available from: https://ec.europa.eu/health//sites/health/files/preparedness_response/docs/ev_20150430_co06_en.pdf. Accessed October 25, 2017.

25. Papastergiou J, Folkins C, Li W, Zervas J. Community pharmacistadministered influenza immunization improves patient access to vaccination. Can Pharm J. 2014;147(6):359-365.

26. Bounthayong M, Christopher ML, Mendes MA, et al. Measuring patient satisfaction in the pharmacy specialty immunization clinic: a pharmacist-run Immunization Clinic at the Veterans Affairs San Diego Healthcare System. Int J Pharm Pract. 2010;18(2):100-107.

27. Drozd EM, Miller L, Johnsrud M. Impact of pharmacist immunization authority on seasonal influenza immunization rates across states. Clin Ther. 2017;39(8):1563-1580.
28. Baroy J, Chung D, Frisch R, et al. The impact of pharmacist immunization programs on adult immunization rates: a systematic review and meta-analysis. J Am Pharm Assoc. 2016;56(4):418-426.

29. Buchan SA, Rosella LC, Finkelstein M, et al. Impact of pharmacist administration of influenza vaccines on uptake in Canada. Can Med Assoc J. 2017;189(4):E146-E152.

30. Pearson G, Yuksel N, Card, D. et al. An information paper on pharmacist prescribing within a health care facility. Can J Hosp Pharm. 2002;55(1):56-62.

31. Tran D, Gatewood S, Moczygemba LR, et al. Evaluating health outcomes following a pharmacist-provided comprehensive pretravel health clinic in a supermarket pharmacy. J Am Pharm Assoc. 2015; 55(2):143-152.

32. Jackson AB, Humphries TL, Nelson KM, Helling DK. Clinical pharmacy travel medicine services: A new frontier. Ann Pharmacother. 2004;38:2160-2165.

33. Hess KM, Dai C, Garner B, Law AV. Measuring outcomes of a pharmacist-run travel health clinic located in an independent community pharmacy. J Am Pharm Assoc. 2010;50:174-180.

34. Houle SKD, Bascom CS, Rosenthal MM. Clinical outcomes and satisfaction with a pharmacist-managed travel clinic in Alberta, Canada. Trav Med Infect Dis. Epub 2018 Mar 1.

35. Steffen R, Lobel HO, Haworth J, Bradley DJ, editors. Travel Medicine. Proceedings of the First Conference on International Travel Medicine, Zurich, Switzerland, 5-8 April 1988. Berlin: Springer-Verlag; 1989.

36. Foong EA, Edwards DJ, Houle S, Grindrod KA. Ready or not? Pharmacist perceptions of a changing scope of practice before it happens. Can Pharm J. 2017;150(6):387-396.

37. International Society of Travel Medicine. ISTM Certificate of knowledge. Available from: http://www.istm.org/certificateofknowledge. Accessed November 3, 2017.

38. Habicht D, Ng S, Dunford D, et al. Incorporating assessment and prescribing for ambulatory ailments skills into practice: an environmental scan of continuing education for pharmacist prescribing in Canada. Can Pharm J. 2017;150(5):316-325.

39. Pharmaceutical Services Negotiating Committee. PSNC Briefing 044/17: Analysis of Minor Ailment Services Data, July 2017. Available from: http://psnc.org.uk/wp-content/uploads/2017/07/PSNC-Briefing044.17-Analysis-of-Minor-Ailment-Services-Data.pdf. Accessed November 3, 2017.

40. Paudyal V, Watson MC, Sach T, et al. Are pharmacy-based minor ailment schemes a substitute for other service providers? A systematic review. Br J Gen Pract. 2013;63(612):e472-e481.

41. Alberta College of Pharmacists. Additional prescribing authorization. Available from: https://pharmacists.ab.ca/additional-prescribingauthorization. Accessed November 4, 2017.

42. College of Pharmacists of Manitoba. Expanded scope of practice extended practice pharmacist. Available from: https://mpha.in1touch org/site/expandedscopeofpractice. Accessed November 4, 2017.

43. Houle SKD, Grindrod KA, Chatterley T, Tsuyuki RT. Publicly funded remuneration for the administration of injections by pharmacists: an international review. Can Pharm J. 2013;146(6):353-364.

44. Centers for Disease Control and Prevention. Yellow fever vaccine. Available from: https://www.cdc.gov/yellowfever/vaccine/index.html. Accessed November 5, 2017.

45. Canada Health Infoway. Immunization Registry. Available from: https:// www.infoway-inforoute.ca/en/component/content/article?id=241. Accessed November 5, 2017.

46. Houle SKD, Atkinson K, Paradis M, Wilson K. CANImmunize: a digital tool to help patients manage their immunizations. Can Pharm J. 2017;150(4):236-238.

47. McInnes RJ, Williamson LM, Morrison A. Unintentional injury during foreign travel: a review. J Travel Med. 2002;9(6):297-307.

48. Hargarten SW, Baker TD, Guptill K. Overseas fatalities of United States citizen travelers: an analysis of deaths related to international travel. Ann Emerg Med. 1991;20(6):622-626.

49. MacPherson DW, Guerillot F, Streiner DL, et al. Death and dying abroad: the Canadian experience. J Trav Med. 2000;7(5):227-233. 
Integrated Pharmacy Research and Practice

Dovepress

\section{Publish your work in this journal}

Integrated Pharmacy Research and Practice is an international, peer-reviewed, open access, online journal, publishing original research, reports, reviews and commentaries on all areas of academic and professional pharmacy practice. This journal aims to represent the academic output of pharmacists and pharmacy practice with particular focus on integrated care. All papers are carefully

peer reviewed to ensure the highest standards as well as ensuring that we are informing and stimulating pharmaceutical professionals. The manuscript management system is completely online and includes a very quick and fair peer-review system, which is all easy to use. Visit http://www.dovepress.com/ testimonials.php to read real quotes from published authors.

Submit your manuscript here: http://www.dovepress.com/integrated-pharmacy-research-and-practice-journal 